\title{
A ENFERMAGEM E O IDOSO PORTADOR DE DEMÊNCIA TIPO ALZHEIMER: desafios do cuidar no novo milênio ${ }^{1}$
}

\author{
Marlene Teda Pelzer ${ }^{2}$
}

\section{Resumo}

Este artigo mostra a associação entre envelhecimento populacional e a demência tipo Alzheimer, considerada como um dos maiores problemas de saúde pública na atualidade. A família tem sido desafiada a assumir o cuidado do idoso fragilizado e dependente, requerendo suporte tanto por parte da enfermeira quanto de organizações não governamentais para poder enfrentar as demandas diárias do cuidar. A enfermagem brasileira precisa organizar seu corpo de conhecimento para fazer frente à complexa dinâmica do cuidado ao idoso que vivencia um processo de demência, oferecendo novas e efetivas estratégias de intervenção.

Palavras-Chave: Demência; Família; Enfermagem Geriátrica; Cuidado Domiciliar.

\begin{abstract}
An association is presented between populational aging and the Alzheimer kind of dementia, considered to be one of the most relevant public health problems of our days. The family, in its challenge to take over the care of the frail dependent elderly, requires the support both of the nurse and of non-governmental organizations (NGOs), so as to be able to cope with the daily demands of care. Brazilian nursing has to organize its knowledge assets so as to face the complex dynamics of caring for the aged who lives through a dementia process. In this process, knew and effective intervention strategies must be implemented.

\footnotetext{
${ }^{1}$ Artigo elaborado na Disciplina Processo de Construção do Conhecimento em Saúde e Enfermagem (2001/2), no Curso de Doutorado em Enfermagem da Universidade Federal de Santa Catarina (UFSC), sob orientação das Profas. Alacoque Lorenzini Erdman e Marta Lenise Prado.

${ }^{2}$ Enfermeira. Professora Adjunto IV do Departamento de Enfermagem da Fundação Universidade Federal do Rio Grande (FURG). Especialista em Gerontologia pela Sociedade Brasileira de Geriatria e Gerontologia (SBGG), Mestre em Assistência de Enfermagem, Doutoranda em Enfermagem pela UFSC. E-mail: pelzer@mikrus.com.br
} 
Keywords: Dementia; Family; Geriatric Nursing; Homecare.

\section{Introdução}

A demência, junto com a incontinência urinária, instabilidade postural e quedas, delírio e depressão, constituem-se nos "gigantes da geriatria" (GORDILHO et alii., 2000), assim identificados por serem considerados eventos típicos e complexos da terceira idade, além de apresentarem alta taxa de prevalência.

Demência é um termo amplo que pode incluir muitas desordens específicas, uma série de definições clínicas para comprovação de casos em estudos epidemiológicos. Ao lado dos transtornos cardiovasculares, as demências são as doenças mais prevalentes acima de 65 anos (HENDRIE et alii., 1997).

As demências enquanto "[. . .] síndrome do dano cognitivo persistente em adultos." (GREEN, 2001, p. 15), representam um significativo problema de saúde pública pela longa extensão e complexidade de manifestações funcionais, emocionais e conseqüências sociais, tanto para a pessoa idosa afetada quanto para seus familiares cuidadores. A doença de Alzheimer (DA) é a causa mais comum de demência em idosos. A DA a partir da década de 70 transformou-se em uma das doenças mais freqüentes e que mais preocupações têm despertado tanto na população quanto na comunidade científica. Isto se deve pela ampliação do seu conceito neste período e pelo significativo envelhecimento da população mundial (NITRINI, 1999). Os sintomas cognitivos mais freqüentes incluem perda de memória, prejuízo da atenção e distúrbios da linguagem (FERRES; KLUGER, 1997).

Nos Estados Unidos, país com significativo aporte de recursos em pesquisas nesta área, aproximadamente 100 bilhões de dólares são gastos anualmente com a doença de Alzheimer (SNOW, 1997), sendo a maior parte deste custo decorrente da internação dos portadores.

Estimativas indicam que uma em cada dez famílias americanas possui um membro com DA e que sua prevalência é de 4,5 milhões de indivíduos afetados, podendo alcançar quatorze milhões na metade do século XXI (NADLER-MOODIE; WILSON,1998; BROOKMEYER; GRAY; KAWAS, 1998), constituindo-se na quarta causa de morte. No 
Canadá, $8 \%$ da população com 65 anos ou mais e 34,5\% com 85 anos ou mais tem diagnóstico de demência.

Conforme Jorm ${ }^{3}$ apud Nitrini, a prevalência de demência aumenta significativamente com a idade, dobrando a cada cinco anos, aproximadamente, a partir dos 65 anos.

Pelo impacto que tem sobre a qualidade de vida tanto dos portadores quanto dos familiares cuidadores, é considerada como um dos maiores problemas de saúde pública da atualidade.

No Brasil, são poucos os estudos epidemiológicos realizados em pessoas com 60 anos ou mais em relação à prevalência da DA, não se dispondo dados atualizados de abrangência nacional. Mais recentemente, alguns estudos como o de Nitrini et alii. (1995), começaram a ser realizados, em amostra de 100 pacientes, não permitindo generalizar os resultados. Neste estudo, a demência tipo Alzheimer representa 54\% da etiologia dos casos de demência.

Engelhardt et alii. (1998) realizaram rastreamento cognitivo de 292 idosos institucionalizados no Rio de Janeiro, utilizando o Mini-exame do Estado Mental com escores de corte diferenciados em função de escolaridade e constataram comprometimento cognitivo em 52,4\%.

Estudos de incidência, por sua vez, fornecem dados mais precisos, mas são mais difíceis de realizar porque exigem a fase inicial de avaliação e pelo menos uma reavaliação alguns anos mais tarde. Ramos et alii. (1998) têm acompanhado uma coorte de 1667 idosos vivendo na comunidade em São Paulo, mas seus resultados ainda não foram registrados.

A trajetória da doença na demência do tipo Alzheimer é representada por um declínio, com perdas ocasionais de estabilidade, levando inevitavelmente a uma profunda deterioração cognitiva e finalmente à morte. Por ser uma doença progressiva, envolve uma série de perdas nas quais os membros da família antecipam e sofrem com cada marco na sua evolução. As incertezas a respeito da trajetória da doença empurram as famílias para se reorganizarem sem o membro afetado ou para minimizarem as demandas da doença, esperando fantasiosamente que o membro doente mantenha suas responsabilidades familiares normais.

3 JORM, A. F. The Epidemiology of Alzheimer's Disease and Related Disorders. London: Chapman and Hall, 1990. Apud Nitrini, 1999, p. 263. 
Dessa forma, a DA pode representar um longo "goodbay" para a família, assim como um luto antecipado e prolongado.

Nas próximas décadas, o número de idosos portadores de demência do tipo Alzheimer (DA) irá aumentar consideravelmente no Brasil, uma vez que a população brasileira está envelhecendo também, e rapidamente.

O tema envelhecimento vem adquirindo, cada vez mais, importância primordial. Desde os anos 89, a velhice e as questões relacionadas com o envelhecimento ocupam cada vez mais espaço entre os temas que preocupam a sociedade brasileira. $\mathrm{O}$ processo de envelhecimento que começa a despontar e que caminha no sentido de uma intensificação, surge no bojo de uma crise econômica, na qual as desigualdades e os problemas sociais parecem se agudizar.

Neste contexto, em que coexistem problemas prementes relacionados com outros segmentos etários, torna-se difícil sustentar um diagnóstico otimista quanto à evolução da capacidade de dar respostas adequadas às crescentes demandas da população idosa. Nem a família, nem a comunidade e muito menos o poder público parecem estar suficientemente preparados para essa nova realidade demográfica. Ao contrário, como afirma Soares et alii. (1992, p. 25-38) no Brasil, “[. . .] o processo de modernização vem acabando com os mecanismos tradicionais de amparo à velhice sem ter ainda conseguido desenvolver mecanismos novos de proteção social típicos do Estado de Bem-Estar prevalentes nas regiões mais desenvolvidas."

Prevê-se que no ano 2025, o Brasil será o sexto país do mundo em população idosa. A população idosa em anos mais recentes (década de 1980), enquanto estrato de consumo e grupo social, começou a ser alvo de ações específicas de saúde no Brasil, embora até o momento com programas ainda incipientes em termos de implantação e experiência acumulada. A população que envelhece cobra assim novas exigências para os serviços sociais e de saúde, de natureza um tanto complexa.

À medida que o número de idosos aumenta na população é necessário um conhecimento maior de suas necessidades e acompanhamento de suas mudanças. A consequiência é a necessidade de informação atualizada no campo da demografia, das mudanças do entorno familiar, social, econômico e de saúde deste segmento. 
A magnitude populacional e o perfil de polimorbidade-mortalidade exigem novas estratégias para fazer frente ao aumento exponencial do número de idosos potencialmente dependentes. Tanto as doenças físicas quanto as mentais podem levar à dependência e, conseqüentemente, à perda da capacidade funcional. A dependência física assim como a mental constituem fatores de risco significativos para a mortalidade, mais relevantes até que as próprias doenças que levaram à dependência, visto que nem todo doente torna-se dependente, conforme revelam estudos populacionais de segmentos de idosos residentes em diferentes comunidades (RAMOS et alli, 1993), assim como estudos longitudinais prospectivos (BOULT et alii, 1994).

A mudança no perfil demográfico e epidemiológico acarreta grandes despesas com tratamentos médicos e hospitalares, ao mesmo tempo em que se configura num desafio para o sistema de saúde. O idoso consome mais serviços de saúde, as internações hospitalares são mais freqüentes e o tempo de ocupação do leito é maior do que o de outras faixas etárias.

O predomínio das enfermidades crônicas não transmissíveis nos idosos e da importância crescente de fatores de risco para a saúde, que requerem, complexamente, ações preventivas em diversos níveis, demandam um modelo de atendimento adequado às necessidades específicas desta clientela, diminuindo a sobrecarga ao idoso, sua família e à própria rede de saúde.

Os países diferem grandemente na capacidade de responder a novas necessidades de segmentos populacionais específicos. O envelhecimento, tal qual a saúde, é silencioso, não o percebemos na sua plenitude, na maior parte das vezes apenas o identificamos quando adoecemos. A assistência à saúde de uma forma global e a de enfermagem em particular, não devem e não podem ignorar as dimensões social, econômica, cultural e política do processo de envelhecimento, com risco de adotar apenas medidas assistenciais paliativas, sem se preocupar em buscar formas de minimizá-las.

Neste sentido, a informação correta e o conhecimento prévio das mudanças são de grande importância para um planejamento efetivo e para avaliação dos serviços que atendem este segmento. 
Como no Brasil o paradigma referente ao manejo das demências é o paradigma cognitivo-cerebral, que valoriza as funções cognitivas, com um referencial mecanicista, no qual o modelo biomédico supervaloriza marcadores genéticos e bioquímicos. Este contexto acaba refletindo sobre como o idoso portador de DA e seus respectivos familiares são assistidos pelos serviços de saúde.

A promoção da qualidade de vida do idoso dependente - em estágios avançados de DA - e dos que o estão cuidando, é uma meta a ser alcançada, desafiando os profissionais e todos aqueles que estão engajados no processo.

O conjunto de conhecimentos e técnicas já produzidos e acumulados, na direção da construção do objeto efetivo do trabalho em saúde com o idoso portador de DA e sua família, é ainda extremamente pequeno, pouco testado e, por isso mesmo, certamente instável, isto é, tendente a uma rápida re-elaboração.

Ensaia-se, aqui, uma abordagem preliminar do problema, com uma formulação ainda em construção de um “ novo" objeto das práticas de saúde relacionadas ao idoso portador de demência tipo Alzheimer e sua respectiva família no contexto da sociedade brasileira contemporânea.

\section{A Família, a Enfermagem e a Sociedade no Contexto da Doença de Alzheimer}

A família contemporânea tem sido desafiada por economistas, técnicos e legisladores a assumir o cuidado como a melhor maneira e a mais barata, de cuidar dos idosos considerados fragilizados ${ }^{1}$ e dependentes. Os estudos sobre a família brasileira e o trabalho social têm-se expandido na última década (CARVALHO, 1994; FERRARI; KALOUSTIAN, 1994). Nesses estudos têm-se ressaltado a existência de algumas famílias que vivem situações especiais de risco que as tornam incapazes de articular minimamente os cuidados de seus membros, necessitando atenção diferenciada do Estado para garantir os direitos de cidadania dos seus membros, como os idosos, por exemplo.

Ao lidar com a doença crônica de um de seus membros ou qualquer outro evento estressante e disruptivo - como alcoolismo, AIDS, demência, entre outros - a família elabora seu próprio plano de inter- 
venção para fazer frente às necessidades de determinado membro que apresenta problemas.

A atenção hoje prestada à família é ainda conservadora e pouco eficiente porque está presa a uma cultura tutelar. Vários municípios brasileiros (São Paulo, Santos e Porto Alegre, entre outros) já possuem programas de acompanhamento e apoio a famílias em situação de crise que colocam em risco a vida de seus membros. Nenhum destes programas porém contempla especificamente a clientela idosa portadora de DA. O mesmo vale para o Programa de Saúde da Família (PSF), lançado em 1993 pelo Ministério da Saúde, com ênfase no cuidado comunitário e que somente em anos recentes tem incorporado a atenção ao idoso, ainda restrita a alguns municípios e algumas regiões.

A promulgação de leis como a de $n^{0} 8.842$, de janeiro de 1994, que dispõe toda a política social direcionada à população idosa (COSTA, 1996), representa um passo importante no sentido de reconhecer a importância desse segmento e de incluí-lo nas políticas públicas.

A Política Nacional de Saúde do Idoso (PNSI) - Portaria Ministério da Saúde n ${ }^{\circ}$ 1.395, de1999, instrumento de que o setor saúde passa a dispor tem como propósito basilar a promoção do envelhecimento saudável, a preservação e/ou a melhoria, ao máximo possível, da capacidade funcional dos idosos, a prevenção de doenças, a recuperação da saúde daqueles que adoecem e a reabilitação daqueles que venham a ter a sua capacidade funcional restringida, de modo a garantir-lhes permanência no meio em que vivem, exercendo de forma independente suas funções na sociedade (GORDILHO et alii, 2000, p. 24).

Há um evidente descompasso no entanto entre a PNSI e a assistência oferecida à população na rede básica de saúde, cujos profissionais além de não priorizarem a atenção ao usuário idoso (atendimento qualificado), não possuem em sua grande maioria preparo específico na área de geriatria/gerontologia (atendimento especializado). A disponibilidade dos serviços não é bem distribuída, fazendo com que muitos idosos recebam primeiros cuidados somente nos estágios mais avançados de suas doenças.

Observa-se no país um dilema em termos de política de saúde para atender às necessidades tanto das faixas etárias jovem quanto idosa, ambas carentes de recursos e de grande demanda de serviços. 
A única organização não governamental voltada especificamente para a Doença de Alzheimer no Brasil é a ABRAz - Associação Brasileira de Alzheimer e Idosos de Alta Dependência, criada em 1993, com regionais na maioria dos estados e que procura dar apoio aos familiares.

Em vários países desenvolvidos, como a Grã-Bretanha, Escócia e Suécia, entre outros, há uma tradição de organizações voluntárias que prestam auxílio aos idosos, inclusive aqueles portadores de demências. Na Escócia foi implantada em 1997 (CLAVEIROLE; MITCHELL; WHITE, 2001), uma rede de enfermagem com enfoque na família, que tem como objetivo oferecer suporte ao uso da enfermagem de família tanto na prática, na pesquisa quanto na educação.

A maioria das universidades brasileiras ainda não está sintonizada com o atual processo de transição demográfica, sendo que o modelo clássico de prevenção que é ensinado nas escolas de saúde pública somente se aplica em sua totalidade para as faixas etárias mais jovens. Há uma escassez de recursos técnicos e humanos necessários para enfrentar a explosão demográfica desse grupo no novo milênio. A própria PNSI explicita a capacitação de pessoal para o planejamento, coordenação e avaliação de ações como imprescindível no âmbito do setor saúde. No Fórum Gaúcho das Instituições de Ensino Superior com Ações Voltadas ao Envelhecimento, transcorrido em setembro de 2000 na PUCRS, foi acordado que a formação especializada em nível do terceiro grau é fundamental. Neste sentido, alguns cursos de Enfermagem estão implantando tópicos específicos relacionados à temática do envelhecimento humano, contribuindo dessa forma para a construção de referenciais que orientem a formação da enfermeira também para a área do idoso.

A população idosa demanda atenção e cuidado por parte da enfermeira nos diferentes contextos em que se encontra, quer na clínica de agudos ou crônicos, em instituições asilares ou na família. Gonçalves et alii. (1996) pontuam que o cuidado humano de modo abrangente e integral representa a essência da prática de enfermagem. Autores como Lynch e Tiedje (1991) e Zerwekh (1992) apontam de maneira expressiva a necessidade da enfermeira aproximar-se do referencial da família para que a comunicação seja efetiva, e a satisfação profissional atingida, indicando modelos conceituais para a intervenção com famílias em situação de risco. 
Observa-se, no país, que a prática assistencial de enfermagem ainda está voltada predominantemente para o segmento materno-infantil, adulto jovem e maduro. Sua formação acadêmica orienta para uma visão curativa e individual de saúde. É preciso romper com a hegemonia da formação tecnicista, onde se valoriza a tecnologia de ponta e buscar formas para humanizar e politizar os profissionais de enfermagem. É imperativo para a enfermagem brasileira engajar-se com a assistência à população idosa, buscando tornar-se "expert" também na área do envelhecimento humano.

Em nosso meio, Elsen (1994), refere que a prática do cuidar de famílias continua permeada por incertezas, apesar da família ter sido sempre considerada como cliente da enfermeira. Segundo a autora, faltam instrumentos precisos para abordar a família e a própria definição de saúde da família ainda não é tida como um consenso.

Com o rápido envelhecimento populacional e o aumento progressivo da prevalência da DA (NITRINI, 1999), a sociedade brasileira irá necessitar de enfermeiras que prestem assistência aos idosos com déficits cognitivos e distúrbios comportamentais tanto no cenário domiciliar, institucional e comunitário, que sejam capazes de prover suporte para os respectivos familiares cuidadores.

Ao envolver-se com o cuidado ao idoso com demência que ainda permanece no domicílio ou que retornou recentemente de uma hospitalização e que pode estar em regime de internação domiciliar, a enfermeira se defronta com aquele familiar que tomou para si a tarefa de desempenhar a função de familiar cuidador. Ao lançar um olhar específico para este contexto reconhecemos a complexidade e singularidade do atendimento domiciliário, que recai sobre um cuidador único na família, identificado como cuidador leigo, informal ou não profissional.

No complexo sistema da doença de Alzheimer, familiares cuidadores podem ser visualizados como co-clientes, assim como cotrabalhadores. Enquanto co-clientes, requerem por parte da enfermeira atenção, cuidado e suporte profissional, pois estão sujeitos a um significativo estresse tanto psicológico quanto físico, tendo sua qualidade de vida bastante alterada. Enquanto co-trabalhadores, requerem suporte profissional para implementar as decisões chaves que terão que tomar ao longo da trajetória da DA; uma parceria terapêutica com a enfermei- 
ra; compartilhamento nas decisões técnicas, viabilizando, assim, uma prática gerontológica humanizada.

Uma busca ao nível da literatura internacional (BANKS,1999; HARVATH et alii.,1994; NOLAN; DELLASEGA, 2000), mostra a crescente atenção em anos recentes à interação entre terapeutas e familiares cuidadores, com um número emergente de modelos para a prática.

Hasselkus (1994) por exemplo, sugere que nos estágios agudos da doença, o profissional assuma a liderança e à medida que a alta hospitalar se aproxima, os cuidadores se tornem mais envolvidos no cuidado, adquirindo confiança e experiência. Assim o papel do profissional ao final deverá mudar para o de facilitador e capacitador.

Em investigação conduzida na Suécia, junto a cuidadores familiares e profissionais acerca do que consideram ser o melhor cuidado para idosos em processo demencial, Ericson et alii. (2001) obtiveram resultados que sugerem que ambos os grupos de cuidadores precisam trabalhar muito unidos caso se deseje que o cuidado centrado na pessoa se torne realidade.

Nolan (2001) enfatiza a necessidade de "pensar no cuidador" e de ser proativo em oferecer informações e treinamento suficiente, de tal maneira que membros familiares se sintam confiantes nas suas habilidades para prover o padrão de cuidado que eles gostariam de oferecer.

Não há no Brasil ao nível da rede pública um programa de atendimento específico ao idoso portador de demência e/ou transtornos cognitivos, com equipe treinada para aconselhar, dar sugestões sobre como administrar os problemas e ajudar a obter acesso a outros tipos de apoios em termos sociais e de saúde. A ausência de esquemas organizados de atendimento domiciliar certamente sobrecarrega a família, pondo em risco o bem-estar e a qualidade de vida não só do idoso, mas de todos os membros.

No contexto de idosos portadores de demência isto é particularmente relevante, uma vez que pesquisas sinalizam para o alto nível de estresse entre cuidadores (ALMBERG; GRAFSTROM; WINBLAD ${ }^{4}$, 1997 apud ERICSON, 2001).

${ }^{4}$ ALMBERG, B.; GRAFSTROM, M.; WINBLAD, B. Caring for a Demented Elderly Person: burden and burnout among caregiving relatives. Journal of Advanced Nursing, Oxford, v. 25, p. 109-116, 1997. Apud ERICSON, 2001, p. 129. 
Nos países industrializados uma nova visão de sistema de cuidado apropriado e abrangente, que enfatiza o cuidado informal, não pago por parte da família e/ou amigos como uma alternativa de baixo custo para a medicina "high-tech" ou tecnologizada, tem emergido nos anos 90.

$\mathrm{O}$ cuidado informal dirigido às pessoas idosas tem recebido crescente reconhecimento (CHAPPELL, 1993), constituindo-se na fonte predominante de cuidado, compreendendo 80 a $90 \%$ dos idosos que permanecem no domicílio (BRODY, 1985).

O sistema de cuidado no Brasil terá que mudar na prática, como mudou em outros países do mundo (BECK; CHUMBLER, 1997), desenvolvendo esquemas que tragam benefícios evidentes tanto para as pessoas idosas quanto para suas famílias. Além disto, terão que ser adequadamente reconhecidos pelas autoridades da área de saúde, como ocorre nos países industrializados.

Ao re-situar a cuidado de enfermagem propriamente dito no interior de uma assistência humanizada, articulada e com contextos sociais e ambientais, bem como ao re-situar os problemas e as questões apresentadas pelos idosos portadores de DA no âmbito de suas famílias, a enfermeira estará incorporando novos saberes e novas formas de enfrentamento.

Em nosso país não existem estudos de abrangência local e/ou regional que reflitam a real prevalência de idosos que se encontram nesta situação. As poucas informações disponíveis são fragmentadas e incompletas. Esta situação aumenta a relevância de estudos capazes de apoiar o estabelecimento de programas de suporte ao cuidado familiar domiciliar do idoso com DA, identificando fatores positivos e negativos do cuidar.

Os dados obtidos a partir destes estudos irão prover o sistema de saúde e a enfermagem gerontogeriátrica em particular, com um entendimento mais compreensivo do papel do cuidador, realçando o desenvolvimento de estratégias de intervenção efetivas para aliviar os efeitos deletérios e realçar dimensões positivas do cuidar no contexto da doença de Alzheimer. Isto irá otimizar não somente o ambiente - domicílio tanto para o cuidador como para o idoso portador de DA, mas também irá beneficiar este idoso portador em termos de postergar a 
institucionalização como resultado de uma família fortalecida, mais disposta e capaz de suportar os desafios do cuidado domiciliar.

Em última instância, os resultados obtidos irão oferecer elementos acerca do cuidado domiciliar recebido pelo idoso portador de DA. Dessa forma será possível contribuir para a implantação de programas de educação continuada, promoção da saúde e qualidade de vida nesta área, entre outros.

\section{Tecendo os Comentários Finais}

Estamos vivendo num mundo que está ficando grisalho e que requer novas posturas frente à complexidade do fenômeno do envelhecimento humano.

A doença de Alzheimer, enquanto área de conhecimento, se constitui em um desafio para a sociedade contemporânea que ingressa no terceiro milênio com expectativas de um crescimento exponencial da população de 60 anos e mais de idade.

A construção de um conhecimento geral acerca do evento doença de Alzheimer se insere no conhecimento de enfermagem, requerendo novas posturas, novos insights e novas articulações.

A enfermagem, enquanto ciência e disciplina, pode preencher os vazios de conhecimento, elaborando padrões que contemplem a complexidade e a diversidade dos fenômenos de interesse comum, entre eles, a doença de Alzheimer.

Um dos fenômenos centrais na enfermagem - o cuidado humano - faz parte do cotidiano das pessoas, integrado ao seu mundo vivido, expresso de diferentes maneiras de uma cultura para a outra (SANTIN, 1998). A enfermeira através do cuidado de enfermagem, busca inserirse no âmbito multidimensional do ser humano, em uma determinada situação de sua vida, em que este necessita uma atenção específica para cuidar e/ou ser cuidado, em seu processo de ser saudável. Este cuidado pode dar-se tanto em nível da saúde do indivíduo, da saúde da família como da saúde comunitária, acolhendo, assim, todas as dimensões na própria humanidade do ser humano.

As atitudes e métodos através dos quais o corpo de conhecimento é formado com a escolha de diferentes caminhos paradigmáticos, darão 
origem a molduras de trabalho na enfermagem junto aos idosos com DA e suas respectivas famílias. A busca e alcance da excelência científica é imprescindível também nesta área.

Há que se destacar neste contexto, o papel da universidade, como um espaço de produção de conhecimento, catalisadora das demandas sociais emergentes, além de seu papel tradicional na formação de recursos humanos.

A enfermagem brasileira precisa se posicionar frente às atuais tendências do conhecimento científico acerca do evento Alzheimer, comunicando, testando e ampliando novos estudos, replicando seus resultados, enfim, sugerindo novos caminhos.

\section{Referências}

1 BANKS, P. Carer Support: time for a change of direction. London: King's Foundation, 1999.

2 BECK, C.; CHUMBLER, N. Planning for the Future of Long-term Care: consumers providers and purchasers. Journal of Gerontological Nursing, Thorofare, v. 10, n. 8, p. 6-13, 1997.

3 BRODY, E. Parent Care as Normative Family Stress. The Gerontologist, Washington, DC, v. 25, n. 3, p. 19-29, 1985.

4 BROOKMEYER, R.; GRAY, S.; KAWAS, C. Projections of Alzheimer's Disease in the United States and the Public Health Impact of Delaying Disease Onset. American Journal of Public Health, Washington, DC, v.88, p.13371342, 1998.

5 BOULT, C. et alii. Chronic Conditions that Lead to Functional Limitation in the Elderly. Journal of Gerontology, Washington, DC, v. 49, n. 1, p. 28-36, 1994.

6 CARVALHO, M.C.B.de. A Priorização da Família na Agenda da Política Nacional. In: KALOUSTIAN, S. M. (Org.). Família Brasileira, a Base de Tudo. São Paulo: Cortez, 1994. p. 93-108.

7 CHAPPEL, N. L. Implications of Shifting Health Care Policy for Caregiving in Canada. Journal of Aging and Social Policy, Bronx, v. 5, n. 2, p. 39-55, 1993.

8 CLAVEIROLE, A; MITCHELL, R; WHITE, D. Family Nursing Network: Scottish innitiative to support family care. British Journal of Nursing, London, v. 10, n. 17, p. 1142-1147, 2001. 
9 COSTA, L. V. A Política Nacional do Idoso-Perspectiva governamental. In: SEMINÁRIO INTERNACIONAL "ENVELHECIMENTO POPULACIONAL:UMA AGENDA PARA O FINAL DE SÉCULO”, 1996, Brasília. Anais. Brasília, DF: Ministério da Previdência e da Assistência Social, 1996. p. 46-64.

10 ELSEN, I. Desafios da Enfermeira no Cuidado da Família. In: BUB, L. (Coord.). Marcos para a Prática de Enfermagem com Famílias. Florianópolis: Ed. da UFSC, 1994. p. 61-77.

11 ENGELHARDT, E. et alii. Idosos Institucionalizados: rastreamento cognitivo. Revista de Psiquiatria Clínica, São Paulo, v. 25, p. 74-79, 1998.

12 ERICSON, I. et alii. What Constitutes Good Care for People with Dementia? Comparising the views of family and professional caregivers. Texto e Contexto - Enfermagem, Florianópolis, v. 10, n. 2, p. 128-139, mai./ago. 2001.

13 FERRARI, M.; KALOUSTIAN, S. Introdução. In: KALOUSTIAN, S. (Org.). Família Brasileira, a Base de Tudo. São Paulo: Cortez, 1994. p. 11-5. 14 FERRES. S. H.; KLUGER, A. Assessing Cognitive Function in Alzheimer's Disease Research. Alzheimer Disease Associated Disorders, Hagerstown, v. 11, suppl. 6, p. 45-49, 1997.

15 GONÇALVES, L. H. T. et alii. O Cuidado do Idoso Fragilizado e de seus Cuidadores no Contexto Domiciliar. Cogitare Enfermagem, Curitiba, v. 1, n. 2, p. 39-43, 1996

16 GORDILHO, A. et alii. Desafios a Serem Enfrentados no Terceiro Milênio pelo Setor na Atenção ao Idoso. Rio de Janeiro: UnATI, 2000.

17 GREEN, R. Diagnóstico e Tratamento da Doença de Alzheimer e Outras Demências. Rio de Janeiro: EPUC, 2001.

18 HARVATH, T. A. et alii. Establishing Partnerships with Family Caregivers: local and cosmopolitan knowledge. Journal of Gerontological Nursing, Thorofare, v. 20, p. 29-35, 1994.

19 HASSELKUS, B. R. From Hospital to Home: family-professional relationships in geriatric rehabilitation. Gerontology and Geriatric Education, Washington, DC, v. 15, p. 91-100, 1994.

20 HENDRIE, H. C. et alii. The Denting Disorders. Psychiatric Quarterly, New York, NY, v. 68, n. 3, p. 261-279, 1997.

21 LYNCH, I.; THIEDJE, L. B. Working with Multiproblem Families: an intervention model for community health nurses. Public Health Nursing, Malden, v. 8, n. 3, p. 147-153, 1991.

22 NADLER-MOODIE, M.; WILSON, M. F. The Latest in Alzheimer's care. Office Nurse, Montvale, v. 8, p. 10-14, 1998. 
23 NITRINI, R. et alii. Evaluation of 100 Patients with Dementia in São Paulo, Brazil: correlation with socioeconomics status and education. Alzheimer Disease Association Disorders, Hagerstown, v. 9, p. 146-151, 1995.

24 IDEM. Epidemiologia da Doença de Alzheimer no Brasil. Revista de Psiquiatria Clínica, São Paulo, v. 26, n. 5, p. 262-267, 1999. Edição Especial.

25 NOLAN, M. R.; DELLASEGA, C. "I really feel I've let him down": supporting family carers during long-term care placement for elders. Journal of Advance Nursing, Oxford, v. 31, p. 759-67, 2000.

26 IDEM. Working with Family Carers: toward a partnership approach. Reviews in Clinical Gerontology, Cambridge, v. 11, p. 91-97, 2001.

27 BRASIL. Ministério da Saúde. Portaria n. 1.395/99 de 10 de dezembro de 1999, Política Nacional de Saúde do Idoso. Disponível em: <http:/www.ufrgs/ 3idade/portaria1395gm.html>. Acesso em 9 mar. 2002.

28 RAMOS, L. et alii. Two-year Follow-up Study of Elderly Residents in São Paulo, Brazil: methodology and preliminary results. Revista de Saúde Pública, São Paulo, v. 32, p. 397-407, 1998.

29 IDEM. Significance and Management of Disability Among rban Elderly Residents in Brazil. Journal of Cross-Cultural Gerontology, Dordrecht, v. 8, p. 313-323, 1993.

30 SANTIN, S. Cuidado e/ou Conforto: um paradigma para a enfermagem, desenvolvido segundo o costume dos filósofos. Texto e Contexto - Enfermagem. Florianópolis, v. 7, n. 2, p. 11-13, 1998.

31 SNOW, C. Medicare HMOS Develop Plan for Future of Alzheimer's Programming. Modern Health, Boston, v. 26, p. 66-68, 1997.

32 SOARES, A. E. et alii. (Org.). A População Idosa no Brasil: perspectivas e prioridades das políticas governamentais e comunitárias. Belo Horizonte: Fundação João Pinheiro, 1992.

33 ZERWEKH, J. V. Laying the Groundwork for Families, Building Trust and Building Strength. Public Health Nursing, Malden, v. 9, n. 1, p. 15-21, 1992. 